\title{
Comparative Efficacy of Three Commercial Ectoparasiticides against Fleas in Naturally Infested Dogs
}

\author{
Froylán Ibarra-Velarde*, Yolanda Vera-Montenegro, Yazmin Alcala-Canto, Miguel Flores-Ramos, \\ Nelyda Saldaña-Hernández
}

Departamento de Parasitología, Facultad de Medicina Veterinaria y Zootecnia, Universidad Nacional Autónoma de México, Ciudad de México, México

Email: ^ibarraf@unam.mx

How to cite this paper: Ibarra-Velarde, F., Vera-Montenegro, Y., Alcala-Canto, Y., Flores-Ramos, M. and Saldaña-Hernández, N. (2019) Comparative Efficacy of Three Commercial Ectoparasiticides against Fleas in Naturally Infested Dogs. Pharmacology \& Pharmacy, 10, 234-243.

https://doi.org/10.4236/pp.2019.105020

Received: April 6, 2019

Accepted: May 14, 2019

Published: May 17, 2019

Copyright (c) 2019 by author(s) and Scientific Research Publishing Inc. This work is licensed under the Creative Commons Attribution International License (CC BY 4.0).

http://creativecommons.org/licenses/by/4.0/

\section{(c) (i) Open Access}

\begin{abstract}
Fleas that infest pets are considered important parasites of both animals and humans. These insects cause irritation and can also transmit zoonotic diseases. Research has led to a rapid expansion in the development of flea control products. In the face of a market that offers dozens of commercial ectoparasiticides for dogs and cats, pet owners and veterinarians must be provided with evidence to support their decision to select a product to control fleas. To compare the efficacy of three commercially available products against companion animal fleas, a trial was conducted on naturally-infested dogs in order to validate their pulicidal activity. Thirty-two flea-infested dogs with fleas were divided into 4 groups $(n=8)$ for each treatment. Group 1 received one pipette of permethrin as a spot-on dose of $650 \mathrm{mg} / \mathrm{ml}$. The second group received $9.7 \%$ fipronil as a spot-on formulation. Group 3 was treated with a spot-on formulation of permethrin $7.40 \%$ plus piperonyl butoxide at $7.40 \%$. Group 4 remained as the untreated control. Fleas of all experimental dogs were examined and counted on days $0,3,7,14,21$ and 28 to determine the percentage of flea reduction. Results showed a $100 \%$ efficacy for all tested products. Identified species were: Ctenocephalides felis (75.7\%), Ctenocephalides canis (15.9\%) and Pulex irritans (9.5\%). Based on these results, it was concluded that the three anti-flea products evaluated under the conditions of this study, produced an excellent efficacy as from the third day after treatment.
\end{abstract}

\section{Keywords}

Fleas, Dogs, Efficacy, Fipronil, Permethrin, Piperonyl Butoxide 


\section{Introduction}

Despite the discovery and development of newer-generation topical (Spot-on) ectoparasiticides for dogs and cats, practitioners and researchers continue to search for products that provide increased duration and spectrum of activity. Dog and cat fleas, for example, can be a serious source of both animal and human irritation, which has led to a rapid expansion in the development of flea control products [1]. Today, veterinarians can select from several options, including combinations of topical and oral insecticides and acaricides for controlling fleas and other ectoparasites in pets. Combination products with pulicidal activity can increase efficacy by managing variations in parasite susceptibility [2]. The efficacy of topical products that control insect infestations in dogs has been previously demonstrated, for example, the combination of permethrin and fipronil showed a significant acaricidal and insecticidal repellent effect against bites on dogs [3] [4]. This combination has also been proved as safe and effective for the treatment and prevention of Ctenocephalides canis and C. felis [5]. Nonetheless, pyrethroid resistance has been reported due to a single nucleotide substitution in the Vssc gene that encodes the voltage-sensitive sodium channel protein and involves sensitivity to pyrethroids; hence pre-exposure to a cytochrome P450 monooxygenase inhibitor such as piperonyl butoxide, significantly increases the effect of pyrethroids [6].

The present trial aimed to evaluate the effectiveness of three commercial anti-flea products on naturally-infested dogs, compared to untreated dogs, under a controlled environment. These combinations were selected because these products are the most frequently sold compounds against fleas in Mexico, and the veterinarians and pet owners need to have information that supports their efficacy and safety.

\section{Materials and Methods}

\subsection{Experimental Animals}

The work was carried out adhering to the guidelines of the Institutional Committee for Use and Care of Experimental Animals of the institution, according to the Mexican Official Regulation NOM-062-ZOO-1999. The study was performed during the flea season in Mexico, i.e., in late summer 2018. Thirty-two flea-infested dogs out of 124 animals were selected on day- 8 based on the highest count of fleas. Dogs of different ages and breeds that harbored infestations over 50 fleas were included in this trial. They were provided by an animal control center. No medications or vaccinations had been given seven days prior to the initiation of the experimental procedures. After an acclimation period of 8 days, animals were ranked according to the number of parasites and were allocated to study groups. Dogs were fed a commercial, dry, pelleted diet according to their age and size. Water was provided ad libitum. Each animal was individually identified with a numbered tag and a colored collar. 


\subsection{Assessment of Efficacy}

The efficacy of the treatment of flea infestation on dogs was performed according to the World Association for the Advancement of Veterinary Parasitology guidelines [7] [8].

On day 0 (day of treatment) the 32 dogs were divided into four groups of 8 animals each, using a randomized design. Dogs were weighed prior to treatment. Group 1 was treated with one pipette of $65 \%$ permethrin $\left(Z_{i n p a r}{ }^{\circledR}\right.$, Halvet, Mexico), applied once on the skin from the scapulae to the sacral region as a spot-on treatment. Pipette size was selected according to the individual body weight (1 $\mathrm{ml} / 15 \mathrm{~kg} / \mathrm{bw}$ ) and applied following manufacturer's instructions. Group 2 received fipronil at $9.7 \%$ (Frontline $^{\circledR}$, Merial, Mexico) applied similarly as in the first group. Group 3 was treated with $7.40 \%$ permethrin plus $7.40 \%$ piperonyl butoxide (Lomo-pon- $S^{\circledR}$, Lapisa, Mexico) applied once as in the permethrin and fipronil treatment groups. The infested and untreated control group received only water at a volume of $1.0 \mathrm{ml}$ per dog. Fleas were removed and counted by thoroughly combing animals with fine-tooth flea combs for at least 10 minutes. Fleas were considered dead if no movement was observed, thus fleas displaying abnormal movement were considered and counted as live [9]. The whole body of all the experimental animals was combed and carefully examined for flea counts and movement on day 3 to determine the percentage of flea reduction as compared with fleas present in the untreated control, according to the following formula [7] [8]:

Efficacy $=\frac{\text { Arithmetic mean number of flea counts }(\text { Control })-\text { Arithmetic mean of flea counts }(\text { Treated })}{\text { Arithmetic mean of flea counts }(\text { Control })} \times 100$

A removal of fleas was done at day 3 on all dogs. The short-term prophylactic efficacy was measured by re-infesting all animals in each study group on days6, 13, 20 and 27 with a mixed population of 100 fleas $C$. felis and C. canis per dog. All dogs were combed off and fleas were counted on days 14, 21, and 28 after re-infestation. Dogs were observed constantly during and after treatments to record adverse reactions or clinical side effects. After day 28, fleas were removed from the control group to prevent a higher level of infestation that may have caused clinical signs.

\subsection{Statistical Analysis}

The primary efficacy calculations were based on arithmetic mean values with geometric mean values considered secondary. For calculation of geometric means, flea counts for each animal were transformed to the natural logarithm of (count +1$)$. Differences between treated and control groups were analysed statistically by an analysis of variance (ANOVA) with a treatment effect on both untransformed and logarithmic transformed data. For flea counts, a significance level of 0.05 was used and all testing was two-sided, in a two-step procedure; pair-wise comparisons were made only if the overall test of treatments was significant at a $\mathrm{p}$ value of 0.05 . 


\section{Results and Discussion}

Dogs treated with each of the three products had a more significant $(\mathrm{p}<0.05)$ reduction of fleas compared with the untreated controls at each re-infestation through day 28 and a $100 \%$ anti-flea efficacy was achieved from day 3 until the end of the study, in contrast to untreated controls, which remained infested throughout the experiment. It was clearly seen that the observations made on the third day after treatment, showed only a few remaining parts of fleas or fecal detritus, but no eggs or live fleas were recorded during the trial. A 100\% anti-flea efficacy was achieved at the end of the experiment in all the treated groups. Three days after treatments were applied, statistical differences were observed between the treated groups with respect to the untreated control $(\mathrm{p}<0.01)$; yet the statistical analysis showed no difference among the treated groups $(\mathrm{p}<0.01)$ (Table 1).

A comparison made regarding sex and weight on the experimental groups showed no statistical differences among them (Table 2).

Taxonomical identifications carried out on the specimens collected from the untreated control group showed that species of identified fleas were: Ctenocephalides felis (84.08\%) and Ctenocephalides canis (15.92\%). Even though lice treatment was not the aim of the current trial, a light infestation with Trichodectes canis was also recorded on day 3 , yet these insects were completely removed after treatment.

Table 1. Flea counts and percentage reduction after treatment three commercial ectoparasiticides in naturally-infested dogs.

\begin{tabular}{ccccc}
\hline Day & $\begin{array}{c}\text { Untreated Control } \\
(\mathrm{n}=8)\end{array}$ & Permethrin $^{*}$ & Fipronil $^{* *}$ & $\begin{array}{c}\text { Permethrin + Piperonyl } \\
\text { Butoxide }^{* * *}\end{array}$ \\
\hline-8 & 61 & $53.4(0)$ & $54.8(0)$ & $59.5(0)$ \\
0 & 41 & $50.7(0)$ & $50.7(0)$ & $52.8(0)$ \\
3 & 57.3 & $0(100) ¥$ & $0(100) ¥$ & $0(100) ¥$ \\
7 & 44.6 & $0(100) ¥$ & $0(100) ¥$ & $0(100) ¥$ \\
14 & 49.2 & $0(100) ¥$ & $0(100) ¥$ & $0(100) ¥$ \\
21 & 37 & $0(100) ¥$ & $0(100) ¥$ & $0(100) ¥$ \\
28 & 62 & $0(100) ¥$ & $0(100) ¥$ & $0(100) ¥$
\end{tabular}

$¥$ Significantly different from untreated control $(\mathrm{p}<0-0.5) ;{ }^{\star} 65 \% ;{ }^{\star *} 9.7 \% ;{ }^{* * *} 7.4 \%+7.4 \%$.

Table 2. Flea counts related to sex for the experimental groups on day 0 .

\begin{tabular}{cccc}
\hline Group & Male (n) & Female (n) & Total \\
\hline Permethrin & $52.6 \pm 4.2(3)$ & $47.9 \pm 4.1(5)$ & $49.4 \pm 4.1$ \\
Fipronil & $42.1 \pm 5.1(5)$ & $54.2 \pm 5.8(3)$ & $45.5 \pm 4.9$ \\
Permethrin + Piperonyl Butoxide & $55.8 \pm 3.6(4)$ & $47.9 \pm 3.5(4)$ & $51.5 \pm 3.5$ \\
Untreated control & $56.9 \pm 4.4(2)$ & $46.1 \pm 3.8(6)$ & $47.9 \pm 4.1$ \\
\hline
\end{tabular}

${ }^{*}$ Arithmetic mean \pm standard deviation. 
The sale and use of ectoparasiticides for the control of arthropod parasites of domestic animals constitute a major sector of the global animal health market and their control still relies heavily on the use of chemicals of whatever origin.

Since the resistance problem to all chemical insecticides available is becoming more and more evident, new alternatives to control these parasites are prompted. However, the development of new drugs is a tough task mainly due to the high costs to develop a new anti-flea compound, hence pharmaceutical companies are trying to optimize the use of the currently available compounds. Among these products, fipronil, cypermethrin, permethrin and tetramethrin have been proved to be highly effective contact insecticides [10]. Nonetheless, clinical signs of toxicity, such as ataxia and hypersalivation, have been observed in dogs and cats treated with different amounts of a formulation containing these compounds [11].

Most commercially available brands of permethrin "spot-on" products are labeled for "use in dogs only" and may be obtained over-the-counter. Permethrin toxicity usually occurs when the owner applies the dog spot-on product in cats that are in close physical contact with recently treated dogs [11]. Nevertheless, it has been stated that large pets are less severely affected by permethrin-containing products than small-sized dogs, as both body mass and hair density increase poisoning due to the great surface area created by the hair. Moreover, if these pets are accidentally sprayed in the mouth, the risk of poisoning increases 60 150 -fold [12].

Fipronil is a phenylpyrazole compound that blocks the transmissions of signals by the inhibitory neurotransmitter, GABA that is present in arthropods [13] [14]. It has been used worldwide for the treatment and control of flea, mite and tick infestations on dogs and cats [15] [16] [17]. Fipronil is highly lipophilic and diffuses into the sebaceous glands of hair follicles that then act as a reservoir, which confers the compound a long-term protection activity [18]. Fipronil effectiveness on other parasites has been demonstrated, namely, against Sarcoptes scabiei [17] [19], the ear mite Otodectes cynotis [19] [20] [21], as well as other mite species, such as Trombicula and Cheyletiella spp. [22].

The potential benefits of a combination of products are numerous, such as prolonging the duration of activity and broadening the ectoparasiticide spectrum, hence providing a convenient single administration to treat multiple parasites at the same time. However, considering that the present study was aimed solely at determining the efficacy against fleas on naturally infested dogs, the advantage of using a combined formulation of permethrin plus piperonyl butoxide was not demonstrated since other ectoparasites from dogs and cats were not included.

In the present study, no clinical signs were observed after the treatment of dogs with either fipronil or permethrin or permethrin + piperonyl butoxide. Further studies should then be conducted to demonstrate the advantage(s) of this combined formulation.

Previous research [23] [24] determined the therapeutic and residual efficacy of 
a topically applied combination of cyphenothrin (40\%) and pyriproxyfen (2\%) against the tick Haemaphysalis elliptica and the flea Ctenocephalides felis on dogs. The product had a therapeutic efficacy of $83.1 \%$ against $H$. elliptica and $97.5 \%$ against $C$. felis two days after treatment.

In another study, 20 cats and seven dogs living in 16 homes, were topically treated according to label directions with spot-on formulations containing either fipronil $(9.8 \%, \mathrm{w} / \mathrm{w})$ combined with (S)-methoprene $(11.8 \%, \mathrm{w} / \mathrm{w})$ or fipronil $(9.8 \%, w / w)$ combined with (S)-methoprene $(8.8 \%, w / w)$. A single application reduced flea populations by $88.44 \%$, within 7 days [25].

It must be highlighted that in the current trial, all ectoparasiticides proved to $100 \%$ control fleas after three days. Even after re-infestation, no fleas were found on counts performed on day 28. Likewise, no adverse effects observed on adults or puppies; demonstrating that they are safe when administered at the recommended dosing levels. It is important to highlight that no side effects of adverse reactions were observed. Moreover, at the end of the study, the physical appearance and health conditions of the treated dogs improved, as healthy-looking skin and shiny coat were observed. Besides, dogs recovered energy after treatment and remained playful. In contrast, dogs of the untreated group seemed depressed and even aggressive, hence, handling of these animals was difficult before treatment was administered at the end of the experiment. Regarding public and animal health issues, a previous report [26] specified that Ctenocephalides canis and $C$. felis are considered the most important ectoparasites of dogs and cats all over the world and can infest humans. Consequently, it is suggested to strategically treat fleas on pets. It would be a mistake to see the current study as definitive, as one major limitation was findings were not based on an artificial infestation. It is also important to mention that after the original patent of the first approved fipronil formulation expired (Frontline, Merial), several generic products were introduced into the veterinary preventive medicine market worldwide. Considering the importance of flea infestations in veterinary medicine, reports that compare pulicidal efficacy of generic preparations help screen key pharmacodynamic parameters that are required to fully declare a generic drug as efficacious as the reference one.

\section{Conclusion}

It can therefore be concluded from our findings that, under the conditions of the current trial, the three commercially available anti-flea products exerted a high and persistent efficacy during a month in naturally-infested dogs. This study can be a useful tool to ease the selection of an efficacious pulicide for dogs.

\section{Availability of Data and Material}

All datasets are included in this manuscript.

\section{Funding}

The authors kindly thank Laboratorios Lapisa, S.A de C.V., Mexico, for the fi- 
nancial support to carry out this study. The present study was carried out in accordance with the current laws of Mexico.

\section{Acknowledgements}

The authors are grateful to Nelyda Hernández Saldañafor technical assistance.

\section{Contributors}

All listed authors contributed to the study design, carried out the experiments, performed laboratory analysis, collaborated with results interpretation, data analysis, manuscript revision and discussion: Performed statistical data analysis and suggested the experimental design: FIV, YVM, YAC and MFR. Wrote the paper: FIV and YAC with input from YVM Grant funding: FIV. All authors supervised the experimental procedures, read and approved the final version of the manuscript.

\section{Consent for Publication}

Not applicable.

\section{Animal Research}

The work was carried out adhering to the guidelines of the Institutional Committee for Use and Care of Experimental Animals of the institution, according to the Mexican Official Regulation NOM-062-ZOO-1999 and Animal Research: Reporting of In Vivo Experiments guidelines was followed.

\section{Author Details}

Faculty of Veterinary Medicine. National Autonomous University of Mexico. Address: Circuito Exterior s/n. Ciudad Universitaria, Coyoacan. Mexico City, 04510, Mexico.

\section{Conflicts of Interest}

The authors declare that they have no competing interests.

\section{References}

[1] Taylor, M.A. (2001) Recent Developments in Ectoparasiticides. The Veterinary Journal, 161, 253-268. https://www.ncbi.nlm.nih.gov/pubmed/11352483 https://doi.org/10.1053/tvjl.2000.0549

[2] Hayes, B., Schnitzler, B., Wiseman, S. and Snyder, D.E. (2015) Field Evaluation of the Efficacy and Safety of a Combination of Spinosad and Milbemycin Oxime in the Treatment and Prevention of Naturally Acquired Flea Infestations and Treatment of Intestinal Nematode Infections in Dogs in Europe. Veterinary Parasitology, 207, 99-106. https://www.ncbi.nlm.nih.gov/pubmed/25465736 https://doi.org/10.1016/j.vetpar.2014.11.011

[3] Dumont, P., Fankhauser, B., Bouhsira, E., Lienard, E., Jacquiet, P., Beugnet, F., et al. (2015) Repellent and Insecticidal Efficacy of a New Combination of Fipronil and 
Permethrin against the Main Vector of Canine Leishmaniosis in Europe (Phlebotomus perniciosus). Parasites \& Vectors, 8, 49.

https://www.ncbi.nlm.nih.gov/pubmed/25622922 https://doi.org/10.1186/s13071-015-0683-y

[4] Dumont, P., Fourie, J.J., Soll, M. and Beugnet, F. (2015) Repellency, Prevention of Attachment and Acaricidal Efficacy of a New Combination of Fipronil and Permethrin against the Main Vector of Canine Babesiosis in Europe, Dermacentor reticulatus Ticks. Parasites \& Vectors, 8, 50.

https://www.ncbi.nlm.nih.gov/pubmed/25622802

https://doi.org/10.1186/s13071-015-0682-Z

[5] Chatzis, M.K., Psemmas, D., Papadopoulos, E., Navarro, C. and Saridomichelakis, M.N. (2017) A Field Trial of a Fixed Combination of Permethrin and Fipronil (Effitix $\left({ }^{\otimes}\right)$ ) for the Treatment and Prevention of Flea Infestation in Dogs Living with Sheep. Parasites \& Vectors, 10, 212. https://doi.org/10.1186/s13071-017-2145-1 https://www.ncbi.nlm.nih.gov/pubmed/28454576

[6] Guntay, O., Yikilmaz, M.S., Ozaydin, H., Izzetoglu, S. and Suner, A. (2018) Evaluation of Pyrethroid Susceptibility in Culex pipiens of Northern Izmir Province, Turkey. Journal of Arthropod-Borne Diseases, 12, 370-377. https://www.ncbi.nlm.nih.gov/pubmed/30918906

[7] Marchiondo, A.A., Holdsworth, P.A., Green, P., Blagburn, B.L. and Jacobs, D.E. (2007) World Association for the Advancement of Veterinary Parasitology (W.A.A.V.P.) Guidelines for Evaluating the Efficacy of Parasiticides for the Treatment, Prevention and Control of Flea and Tick Infestation on Dogs and Cats. Veterinary Parasitology, 145, 332-344. https://www.ncbi.nlm.nih.gov/pubmed/17140735 https://doi.org/10.1016/j.vetpar.2006.10.028

[8] Marchiondo, A.A., Holdsworth, P.A., Fourie, L.J., Rugg, D., Hellmann, K., Snyder, D.E., et al. (2013) World Association for the Advancement of Veterinary Parasitology (W.A.A.V.P.) Second Edition: Guidelines for Evaluating the Efficacy of Parasiticides for the Treatment, Prevention and Control of Flea and Tick Infestations on Dogs and Cats. Veterinary Parasitology, 194, 84-97.

https://www.ncbi.nlm.nih.gov/pubmed/23741753

https://doi.org/10.1016/j.vetpar.2013.02.003

[9] Snyder, D.E., Rumschlag, A.J., Young, L.M. and Ryan, W.G. (2015) Speed of Flea Knockdown of Spinosad Compared to Afoxolaner, and of Spinosad through 28 Days Post-Treatment in Controlled Laboratory Studies. Parasites \& Vectors, 8, 578. https://www.ncbi.nlm.nih.gov/pubmed/26552479 https://doi.org/10.1186/s13071-015-1195-5

[10] Niazi, A., Goodarzi, M. and Yazdanipour, A.A. (2008) Comparative Study between Least-Squares Support Vector Machines and Partial Least Squares in Simultaneous Spectrophotometric Determination of Cypermethrin, Permethrin and Tetramethrin. Journal of the Brazilian Chemical Society, 19, 536-542. https://doi.org/10.1590/S0103-50532008000300023

[11] Gfeller, R.G. and Messonnier, S.P. (2004) Handbook of Small Animal Toxicology and Poisonings. Second Edition, Mosby, St. Louis.

[12] Jachowski, D.S., Skipper, S. and Gompper, M.E. (2011) Field Evaluation of Imidacloprid as a Systemic Approach to Flea Control in Black-Tailed Prairie Dogs, $C y^{-}$ nomys ludovicianus. Journal of Vector Ecology, 36, 100-107.

https://www.ncbi.nlm.nih.gov/pubmed/21635647 https://doi.org/10.1111/j.1948-7134.2011.00146.x

[13] Rauh, J.J., Lummis, S.C. and Sattelle, D.B. (1990) Pharmacological and Biochemical 
Properties of Insect GABA Receptors. Trends in Pharmacological Sciences, 11, 325-329. https://www.ncbi.nlm.nih.gov/pubmed/2168103 https://doi.org/10.1016/0165-6147(90)90236-2

[14] Raymond-Delpech, V., Matsuda, K., Sattelle, B.M., Rauh, J.J. and Sattelle, D.B. (2005) Ion Channels: Molecular Targets of Neuroactive Insecticides. Invertebrate Neuroscience, 5, 119-133. https://www.ncbi.nlm.nih.gov/pubmed/16172884 https://doi.org/10.1007/s10158-005-0004-9

[15] Postal, J.M.R., Ostal, L., Jeannin, P.C. and Consalvi, P.J. (1995) Field Efficacy of a Mechanical Pump Spray Formulation Containing 0.25\% Fipronil in the Treatment and Control of Flea Infestation and Associated Dermatological Signs in Dogs and Cats. Veterinary Dermatology, 6, 153-158. https://doi.org/10.1111/j.1365-3164.1995.tb00059.x

[16] Franc, M., Lienard, E., Jacquiet, P., Bonneau, S. and Bouhsira, E. (2015) Efficacy of Fipronil Combined with Permethrin Commercial Spot on (Effitix) Preventing Culex pipiens from Feeding on Dogs. Parasitology Research, 114, 2093-2097.

https://www.ncbi.nlm.nih.gov/pubmed/25739921 https://doi.org/10.1007/s00436-015-4397-Z

[17] Curtis, C.F. (1996) Use of 0.25 Percent Fipronil Spray to Treat Sarcoptic Manage in a Litter of Five-Week-Old Puppies. Veterinary Record, 139, 43-44. https://www.ncbi.nlm.nih.gov/pubmed/8839491 https://doi.org/10.1136/vr.139.2.43

[18] Birckel, P., Cochet, P.B. and Weil, A. (1998) Cutaneous Distribution of C14 Fipronil in the Dog and Cat Following a Spot-On Administration. Vol. 3, Butterworth Heinemann, Oxford.

[19] Curtis, C.F. (2004) Current Trends in the Treatment of Sarcoptes, Cheyletiella and Otodectes Mite Infestations in Dogs and Cats. Veterinary Dermatology, 15, 108-114. https://www.ncbi.nlm.nih.gov/pubmed/15030559 https://doi.org/10.1111/j.1365-3164.2004.00362.x

[20] Vincenzi, P. and Gauchi, C. (1997) Efficacy of Fipronil against Ear Mites Otodectes cynotis in Dogs and Cats. Proceedings of the 14th Annual Congress ESVD-ECVD, 5-7 September 1997, 117.

[21] Beugnet, F., Bouhsira, E., Halos, L. and Franc, M. (2014) Preventive Efficacy of a Topical Combination of Fipronil-(S)-Methoprene-Eprinomectin-Praziquantel against Ear Mite (Otodectes cynotis) Infestation of Cats through a Natural Infestation Model. Parasite, 21, 40. https://www.ncbi.nlm.nih.gov/pubmed/25148648 https://doi.org/10.1051/parasite/2014042

[22] Nuttall, T.J., French, A.T., Cheetham, H.C. and Proctor, F.J. (1998) Treatment of Trombicula autumnalis Infestation in Dogs and Cats with a 0.25 Percent Fipronil Pump Spray. Journal of Small Animal Practice, 39, 237-239.

https://www.ncbi.nlm.nih.gov/pubmed/9631359 https://doi.org/10.1111/j.1748-5827.1998.tb03641.x

[23] Fourie, J.J., Fourie, L.J., Horak, I.G. and Snyman, M.G. (2010) The Efficacy of a Topically Applied Combination of Cyphenothrin and Pyriproxyfen against the Southern African Yellow Dog Tick, Haemaphysalis elliptica, and the Cat Flea, Ctenocephalides felis, on Dogs. Journal of the South African Veterinary Association, 81, 33-36. https://www.ncbi.nlm.nih.gov/pubmed/20649152 https://doi.org/10.4102/jsava.v81i1.93

[24] Fourie, J.J., Crafford, D., Horak, I.G. and Stanneck, D. (2013) Prophylactic Treatment of Flea-Infested Dogs with an Imidacloprid/Flumethrin Collar (Seresto ${ }^{\star}$, Bayer) to Preempt Infection with Dipylidium caninum. Parasitology Research, 112, 
33-46. http://www.ncbi.nlm.nih.gov/pubmed/23771717 https://doi.org/10.1007/s00436-013-3279-5

[25] Dryden, M.W., Payne, P.A., Vicki, S., Riggs, B., Davenport, J. and Kobuszewski, D. (2011) Efficacy of Dinotefuran-Pyriproxyfen, Dinotefuran-Pyriproxyfen Permethrin and Fipronil-(S)-Methoprene Topical Spot-On Formulations to Control Flea Populations in Naturally Infested Pets and Private Residences in Tampa, FL. Veterinary Parasitology, 182, 281-286. https://www.ncbi.nlm.nih.gov/pubmed/21705147 https://doi.org/10.1016/j.vetpar.2011.05.054

[26] Halos, L., Beugnet, F., Cardoso, L., Farkas, R., Franc, M., Guillot, J., et al. (2014) Flea Control Failure? Myths and Realities. Trends in Parasitology, 30, 228-233. https://www.ncbi.nlm.nih.gov/pubmed/24661796 https://doi.org/10.1016/j.pt.2014.02.007

\section{Abbreviations}

bw = body weight 